\title{
Buckling Behaviour of Short Cylindrical Functionally Gradient Polymeric Materials
}

\author{
M. U. UysaL ${ }^{a, *}$, M. KREMZER ${ }^{b}$
}

${ }^{a}$ Yildiz Technical University, Department of Mechanical Engineering, 34349, Istanbul, Turkey

${ }^{b}$ Silesian University of Technology, Institute of Engineering Materials and Biomaterials, 44-100, Gliwice, Poland

In the present study, the buckling behavior of short cylindrical Functionally Gradient Polymeric Materials (FGPMs) was studied. Besides, the structure and graphite distributions of the FGPMs were investigated. Epoxy resin was used as polymeric matrix component and two types of graphite powder materials, PAM96/98 and PV60/65, were selected. Graphite powders were added in quantities of 3, 6, 9, and $12 \%$ of volume respectively. Short cylindrical FGPMs samples were manufactured by centrifugal casting method. The structure and graphite distribution of FGPMs samples were investigated by light microscope and image processing program. It was observed that the graphite distribution had varied between the inner and outer diameter. The buckling behavior of short cylindrical FGPMs was analyzed by finite element analysis. The buckling loads of FGPMs samples were predicted.

DOI: $10.12693 /$ APhysPolA.127.1355

PACS: 81.05.Qk

\section{Introduction}

Functional graded materials (FGMs) are innovative composite materials whose composition and microstructure vary in space following a predetermined law. The gradual change in composition and microstructure gives place to a gradient of properties and performances [1]. A typical FGM is an inhomogeneous composite made from different phases of material constituents [2]. In present study, polymeric gradient materials based on epoxy resin filled with graphite were investigated. Spherical or nearly spherical particles are embedded within an isotropic matrix. The different micro-structural phases in FGMs have different function and the overall FGMs attain the multistructural status from their property gradation. By gradually varying the volume fraction of constituent materials, their properties exhibit a smooth and continuous change from one surface to another, thus eliminating interface problems.

Most of early research studies in FGMs mainly focused on thermal stress analysis and fracture mechanics. But recently, many comprehensive surveys for bending and buckling analysis of plate and shell structures made of FGMs were presented [3-8]. Besides, cylindrical shell is one of the important structures used widely in engineering applications. When the cylindrical shells are subjected to compressive loads, external pressure etc., they may be buckled. Shen and Chen [9] studied the buckling and analyses of cylindrical shells under combined loads. Paimushin [10] reported details of local and global buckling of cylindrical shells subjected to compression and external pressure. Shen [11] and Shen et al. [12] presented the buckling analysis of laminated composite cylindrical shells under combined external pressure and

*corresponding author; e-mail: mineuslu@yildiz.edu.tr axial compression. Besides, the investigation of buckling of cylindrical FGMs shells is a necessary fundamental problem, which has attracted attention of many researchers. Khazaeinejad [13] studied the stability of a circular cylindrical shell composed of FGMs with the elastic modulus varying continuously in the thickness direction, under combined axial compression and external pressure. Dung and Hoa [14] obtained the results on the nonlinear buckling and post-buckling analysis of eccentrically stiffened FGM circular cylindrical shells under external pressure. Researches describing functional materials based on polymers or with polymeric matrix are still very limited. In polymers, similar to other materials, the compositional and microstructural gradients are intended to obtain an optimum combination of component properties. There are many methods of forming polymeric gradient materials, which are similar to those of metallic or ceramic FGMs. Polymer modification aims to improving of mechanical characteristics. With the increasing use of FGPMs (functional polymer graded materials), it is important to understand the buckling behavior of functionally graded cylindrical shells.

In this study, the structure and graphite distributions of the FGPMs were investigated. Epoxy resin was used as polymeric matrix component and two types of graphite powder materials, PAM96/98 and PV60/65, were selected as fillers. The aim of this work is to compare polymeric materials, which have different graphite content and to evaluate influence of graphite ratio on the critical buckling load of FGPMs.

\section{Experimental study \\ 2.1. Materials and equipment}

Epoxy resin (Epidian 6) which was cured with Z1 material, both produced by "Organika-Sarzyna" Chemical Plant S.A. (Poland), was used as the polymeric matrix component [15]. Graphite powders, PAM96/98 and PV60/65, produced by Koh-I-Noor (Czech Republic) were used as fillers. The graphite content ratios of 3 , 
6,9 , and 12 vol.\% were chosen. In this research, the centrifugal casting method, being one of the most effective methods for polymeric gradient materials creation, was applied [16, 17]. Dimensions of produced FGPMs can be seen in Fig. 1.

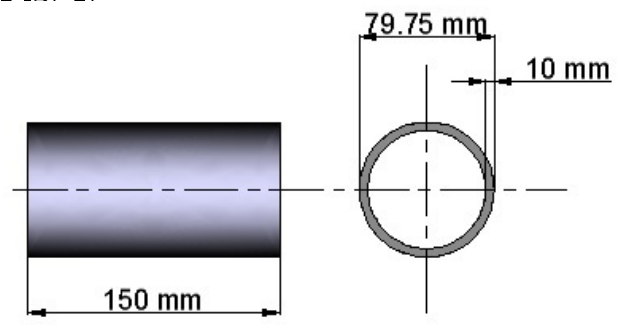

Fig. 1. Dimension of specimens manufactured by centrifugal casting.

The samples, sliced from tubular FGPMs specimen, were wet grinded, polished, and etched in pure ethyl acetate (Poch Co. Gliwice, Poland). Thus prepared samples were observed through the optical microscope. During polishing, samples were cooled by diamond suspension made by Struers Co. Etching time was one minute for each sample, and then all samples were observed in $\times 200$ magnification, using the light microscope LECIA (MEF4A) equipped with Axiovision software. The graphite particles in FGPM structure were detected and the area percentages of the detected elements were calculated by using image analyzer Leica QWin. In Table I, symbols I, II, III, IV, and V represent the five regions from the outside to inside. The percentages of graphite area density in these regions are given in Table I.

The percentages of graphite density ( $\%$ area). TABLE I

\begin{tabular}{c|c|c|c|c|c}
\hline \hline Material \% & I & II & III & IV & V \\
\hline $3 \%$ PAM & 2.1 & 1.8 & 1.6 & 1.1 & 1.0 \\
\hline $3 \%$ PV & 12.0 & 4.2 & 1.2 & 0.2 & 0.0 \\
\hline $6 \%$ PAM & 2.5 & 2.2 & 1.9 & 0.8 & 0.0 \\
\hline $6 \%$ PV & 7.8 & 5.6 & 3.6 & 2.0 & 0.3 \\
\hline $9 \%$ PAM & 1.7 & 0.95 & 0.69 & 0.3 & 0.0 \\
\hline $9 \%$ PV & 10.9 & 6.7 & 6.2 & 2.3 & 0.2 \\
\hline $12 \%$ PAM & 2.6 & 3.3 & 3.0 & 1.8 & 0.3 \\
\hline $12 \%$ PV & 22.1 & 19.2 & 17.0 & 10.0 & 5.0
\end{tabular}

\subsection{Prediction of the modulus of composite with non-spherical fillers}

Several models for predicting the Young's modulus of polymer composites with non-spherical fillers have been proposed in the literature, for instance, Guth model, Brodnyan model, Halpin-Tsai model, LewisNielsen model and Verbeek-Focke model. The HalpinTsai equations [18] are widely used to predict the modulus of unidirectional composites [19, 20] and this model is used in the present study. The Halpin and Tsai equations are a general form of the Kerner equation and many other equations [21]. The modulus of elasticity of FGPMs was determined by

$$
\frac{E_{\text {composite }}}{E_{\text {polymer }}}=\frac{1+\xi \eta \nu_{f}}{1-\eta \nu_{f}},
$$

where $\xi$ is a shape factor that depends on the geometry of the filler particle and $\nu_{f}$ is the vol. $\%$ of filler. The shape factor was assumed as 5.1 due to the fact that graphite shape was flake-like [22]. The parameter $\eta$ is determined by

$$
\eta=\frac{E_{\text {filler }} / E_{\text {polymer }}-1}{E_{\text {filler }} / E_{\text {polymer }}+\xi} .
$$

In this study, the modulus of elasticity was calculated by using Equations 1, 2 and values from Table I for each of the five regions. In calculations, $E_{P A M}, E_{P V}$, and $E_{\text {epoxy }}$ were taken as 1000,1020 , and $3.24 \mathrm{GPa}$, respectively and $\nu_{P A M}, \nu_{P V}$ and $\nu_{\text {epoxy }}$ were taken as 0.261 , 0.272 , and 0.25 , respectively.

\section{Analysis of PAM and PV samples}

The commercial software (ANSYS) has been employed to develop a finite element model of FGPMs cylindrical samples. An external pressure was applied to the FGPM models until the buckling occurred. The presented model has incorporated 2D PLANE elements along an axial cross section, swept across half of the model, to take advantage of symmetry. PLANE25 was used for $2 \mathrm{D}$ modeling of axisymmetric structures with nonaxisymmetric loading (Fig. 2). The element was defined by four nodes, having three degrees of freedom per node: translations in the nodal $x, y$, and $z$ directions. These directions corresponded to the radial, axial, and tangential directions, respectively, for cross section nodal coordinates. The global cartesian $y$-axis was assumed to be the axis of symmetry. Further, the model was developed only in the $+x$ quadrants. Hence, the radial direction was in the $+x$ direction. The numerical model was divided into finite elements, satisfying the equilibrium and compatibility at each node and along the boundaries between the elements. Elastic buckling analysis was used to predict the buckling loads and the corresponding buckling shapes. Since the unit load (1 MPa) was specified, the load factors have represented the critical buckling load in the first mode. Several mode extraction methods are available in ANSYS. The Block Lanczos method, which is recommended for most applications, was used for extraction of three eigenvalues.

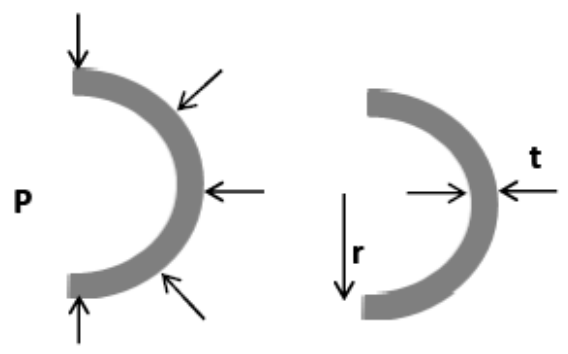

Fig. 2. External pressure buckling of model.

\section{Analysis of results and discussion}

The influences of graphite type and graphite ratio (vol.\%) on the buckling load are presented in Fig. 3, 4. The percentages of graphite volume ratio in FGPMs are compared for first three modes. Figure 3a shows that 
the critical buckling load (mode 1) increases by $9.78 \%$ when the percentage of volume ratio of PAM96/98 is raised from $3 \%$ to $12 \%$. The buckling loads tend to increase when the modes change between 1, 2, and 3, respectively. However, the increase is slight between mode 1 and mode 2 and it is drastic between mode 2 and mode 3 . The maximum change, reflecting the difference of graphite material ratio, is seen in case of 6 vol.\% and 9 vol.\%. The buckling load increases by $11.64 \%$ at PV60/65 volume ratio of $12 \%$, when comparing mode 1 and mode 3 (Fig. 3b).
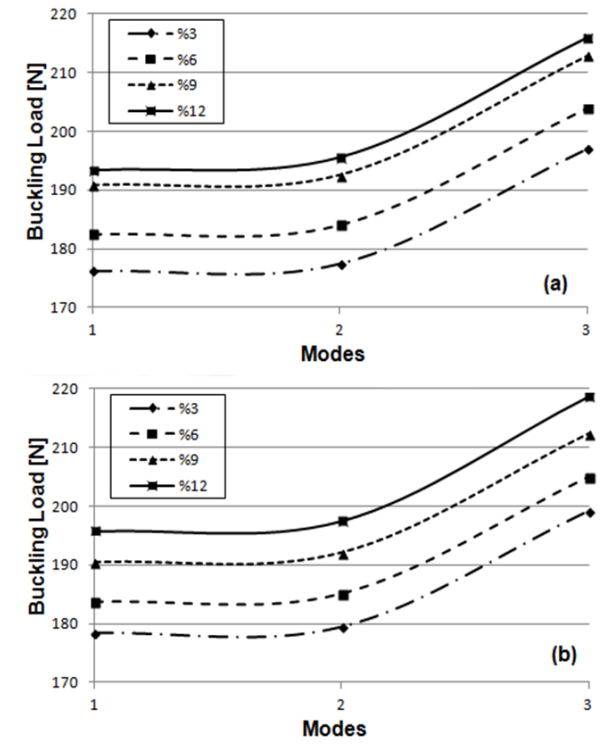

Fig. 3. Effect of the graphite volume ratio on the buckling loads in first three modes a) PAM, b) PV.

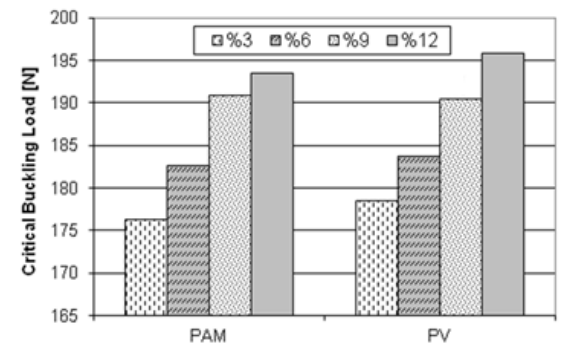

Fig. 4. Effect of the graphite type on the buckling loads.

The critical buckling loads of PAM96/98 and PV60/65 FGPM samples are given in Fig. 4. The results are found to be similar for two different types of graphite. Although same manufacturing method was applied and same graphite ratio was added in two types of materials, the higher critical buckling loads were obtained for PV60/65, with the exception of sample with $9 \%$ volume ratio.

\section{Conclusion}

This study was performed in order to evaluate the percentage area of graphite distribution of two different gradient composites and evaluate the influence of buckling loads on the buckling behavior. The distribution of the reinforcement in the FGPMs is non-homogeneous. This fact causes different material properties. The results showed that the centrifugal casting method was suitable for producing FGPMs with different elastic modulus on the inner and outer side of cylindrical samples. Inside of these composites, continuous gradient of elastic modulus was achieved and it was important to understand the buckling behavior of functionally graded polymeric cylindrical samples. The analysis results showed that PV60/65 graphite generally provided the higher buckling loads than the PAM96/98 graphite. In addition, the buckling loads have increased when the volume ratio of graphite was raised The highest buckling load was obtained in the sample containing 12 vol.\% of PV60/65 graphite.

\section{References}

[1] M. Koizumi, M. Niino, MRS Bull. 20, 19 (1995).

[2] H.M. Yim, L.Z. Sun, G.H. Paulino, Acta Mater. 52, 3535 (2004).

[3] L.H. Yu, C.Y. Wang, AIAA J. 46, 3163 (12).

[4] M. Mohammadi, A.R. Saidi, E. Jomehzadeh, Appl. Comp. Mater. 17, 81 (2010).

[5] V. Birman, L.W. Byrd, Appl. Mech. Rev. 60, 192 (2007).

[6] V. Birman, Compos. Eng. 5, 913 (1995).

[7] E. Feldman, J. Aboudi, Compos. Struct. 38, 29 (1997).

[8] M. Ganapathi, T. Prakash, N. Sundararajan, J. Eng. Mech. 132, 902 (2006).

[9] H.S. Shen, T.Y. Chen, Thin Wall. Struct. 12, 321 (1991).

[10] V.N. Paimushin, Mech. Solid 42, 437 (2007).

[11] H.S. Shen, Thin Wall. Struct. 25, 297 (1996).

[12] H.S. Shen, P. Zhou, T.Y. Chen, Thin Wall. Struct. 15, 43 (1993).

[13] P. Khazaeinejad, M.M. Najafizadeh, J. Jenabi, M.R. Isvandzibaei J. Pressure Vessel Technol. 132, 064501 (2010).

[14] D.V. Dung, L.K. Hoa, Thin Wall. Struct. 63, 117 (2013).

[15] J. Stabik, M. Szczepanik, A. Dybowska, Ł . Suchoń, JAMME 38, 56 (2010).

[16] M. Uslu, M. Kremzer, Int. J. Arts. Sci. 4, 1 (2011).

[17] M. Uslu, M.Sc. Thesis, Polymeric Matrix Composite Materials Reinforced by Graphite, Erasmus Student, Silesian University of Technolog 2010.

[18] J.C. Halpin, J.L. Kardos, Polym. Eng. Sci. 16, 344 (1976).

[19] C.L. Tucher III, E. Liang, Comp. Sci. Technol. 59, 655 (1999).

[20] T.D. Fornes, D.R. Paul, Polymer 44, 4493 (2003).

[21] L.E. Nielsen, R.F. Landel, Mechanical Properties of Polymers and Composites, New York: Marcel Dekker 1994.

[22] W. Mhike, W.W. Focke, J. Vinyl. Addit. Techn. 19, 258 (2013). 